\title{
Effective minority carrier lifetime in double-sided macroporous silicon
}

\author{
V.F. Onyshchenko, L.A. Karachevtseva \\ V. Lashkaryov Institute of Semiconductor Physics, National Academy of Sciences of Ukraine \\ 41, prosp. Nauky, 03680 Kyiv, Ukraine, \\ E-mail: lakar@isp.kiev.ua
}

\begin{abstract}
In this paper, we showed that the effective lifetime of minority charge carriers in double-sided macroporous silicon is determined from a system of two transcendental equations. This system of equations is transformed into a system of equations for determining the effective lifetime of minority charge carriers for monocrystalline silicon in the absence of macropores. The system of equations was found by solving the diffusion equation for minority carriers, recorded for two macroporous layers and a monocrystalline substrate between them. The solution of the nonstationary diffusion equation written for two layers of macroporous silicon and a monocrystalline substrate between them are complemented with boundary conditions on the surfaces of the sample of macroporous silicon and on the boundary between each macroporous layer and monocrystalline substrate. The effective lifetime of minority carriers in the double-sided macroporous silicon depends on such values as: the bulk lifetime of minority carriers, diffusion coefficient of minority carriers, and thickness of the monocrystalline substrate between macroporous layers. In addition, the effective lifetime depends on the values inherent to each macroporous layer: the depth of the macropores, average diameter of macropores, average distance between the centers of macropores, volume fraction of macropores, rate of surface recombination. Effective recombination of excess charge carriers in the doublesided macroporous silicon is defined by recombination of excess charge carriers on the surface of macropores and limited by diffusion of charge carriers from the monocrystalline substrate to recombination surfaces in each macroporous layer. We calculated the effective lifetime of minority carriers in the double-sided macroporous silicon depending on the depth of macropores. We used a numerical method to verify the accuracy of calculations performed using the system of analytical equations, which defines the effective lifetime of minority charge carriers in the double-sided macroporous silicon. The numerical method showed the coincidence of calculations aimed at the effective lifetime of minority carriers. We observed a discrepancy of calculations, when the sum of the depths of macropores in two macroporous layers is close to the thickness of macroporous silicon sample.
\end{abstract}

Keywords: lifetime of minority charge carriers, double-sided macroporous silicon.

https://doi.org/10.15407/spqeo23.01.29

PACS 72.20.Jv, 73.25.+i

Manuscript received 14.11.19; revised version received 16.12.19; accepted for publication 18.03.19; published online 23.03.19.

\section{Introduction}

Macroporous silicon has found application in solar cells [1]. A layer of macroporous silicon is created on a monocrystalline silicon wafer by electrochemical etching. It improves absorption of light [2] and is a broadband anti-reflective coating for silicon solar cells [3]. The relationship between morphology of the macroporous layer and absorption of light is calculated using an analytical model. Due to this, the opto-electrical parameters of the solar cell with layers of macroporous silicon are optimized [4]. Two-dimensional structures of macroporous silicon were coated with layers of microporous silicon, CdTe, surface nanocrystals, and $\mathrm{SiO}_{2}$ nano-coating. The electro-optical effect showed a strong influence of impurity states on the surface of macroporous silicon structure with nano-coating [5]. The coating of porous silicon surface with a thin carbon film 
allowed the significant increase in efficiency and stability of electron emission current [6]. The surface of macroporous silicon is often coated with a layer of silicon oxide, which effectively passivates this surface and improves the effective lifetime of minority charge carriers. The effective lifetime of minority carriers in a passivated layer of macroporous silicon, which is used in solar cells, is simulated in [7]. The effective lifetime of minority carriers in solar cells with a surface structured with cones and pyramids is calculated depending on the diameter of the cone base, side length of the pyramid base, height of the cone and pyramid [8]. The experimentally measured effective lifetime of minority charge carriers in $n$-type black silicon is $1.55 \mathrm{~ms}$. Numerical calculations showed that the surface lifetime determines the effective lifetime. At the same time, the bulk lifetime of minority carriers in polished $p$-type silicon is less than the lifetime on the surface. The photoconductivity of macroporous silicon is investigated. It depends on the diameter, distance between macropores and their depth [9]. It has maxima depending on the angle of incidence of electromagnetic radiation, diameter and average distance between macropores [10]. A diffusion model of photoconductivity relaxation in macroporous silicon is presented in [11]. The photoconductivity relaxation time in macroporous silicon is calculated by the finite differences method. The mechanisms of charge carrier transfer through the surface barrier in electroluminescent structures of porous silicon [12] and structures of macroporous silicon [13] are investigated, too. The charge transfer mechanisms in the space charge region affect the relaxation time of photoconductivity in macroporous silicon. This is confirmed by the temperature dependence of the photoconductivity kinetics, which was measured in macroporous silicon at $80 \ldots 300 \mathrm{~K}[11,13]$. The distribution of excess minority carriers in a layer of macroporous silicon and a monocrystalline substrate is calculated using the finite differences method to calculate the photoconductivity in a layer of macroporous silicon and a monocrystalline substrate [14]. The maxima of distribution inherent to excess minority carriers were detected both in the layer of macroporous silicon and in the monocrystalline substrate. Relaxation of distribution of excess minority carriers in macroporous silicon was also calculated using the finite differences method [15]. A rapid decrease in the maximum of distribution function for excess minority carriers in macroporous layer and a slow decrease in distribution function in a monocrystalline substrate were revealed [15].

The aim of this work is to find the effective lifetime of minority charge carriers in double-sided macroporous silicon, which is defined by the system of two transcendental equations, as well as to identify the relationship between the effective minority carrier lifetime in double-sided macroporous silicon and the characteristics of each macroporous layer, namely: the size of macropores, distance between these macropores, depth of macropores, and the rate of surface recombination.

\section{Effective minority carrier lifetime in double-sided macroporous silicon}

Let us assume that there is no bulk recombination at $t=0$. For the one-dimensional case, the non-stationary diffusion equation for excess minority charge carriers can be written as:

$$
\frac{\partial}{\partial t} \delta p(x, t)=D_{p} \frac{\partial^{2}}{\partial x^{2}} \delta p(x, t)-\frac{\delta p(x, t)}{\tau_{b}},
$$

where $\delta p(x, t)$ is the excess minority carrier concentration, $t$ - time, $x$ - coordinate, $D_{p}$ - minority carrier diffusion coefficient, $\tau_{b}-$ minority carrier bulk lifetime. Boundary conditions look as follows:

$$
g_{s}\left(x_{0}, t\right)-s_{p} \delta p\left(x_{0}, t\right)=e^{-1} j_{p}\left(x_{0}, t\right),
$$

where $e$ is the elementary charge, $\delta p\left(x_{0}, t\right)$ - excess minority carrier concentration on the surface that is perpendicular to the $x$-axis and passes through the point $x_{0}, g_{s}\left(x_{0}, t\right)$ - surface generation rate for excess minority carriers on this surface, $j_{p}\left(x_{0}, t\right)$ - current density of excess minority carriers on this surface, $s_{p}$ - surface recombination velocity of excess minority carriers on this surface. The diffusion current of excess minority carriers $j_{p}$ that flows to the surface is recorded with the minus sign. The general solution of equation (1) is written as:

$$
\delta p(x, t)=\sum_{n=1}^{\infty} A_{n} \exp \left(-\frac{t}{\tau_{n}}\right) \cos \left(n a_{s} x\right),
$$

where $A_{n}$ is the Fourier series expansion coefficients of the initial distribution of excess minority charge carriers, $\tau_{n}$ are characteristic times, $n-$ natural numbers, $a_{s}$ is a constant characteristic number. Between $\tau_{n}$ and $a_{s}$, there is a relationship:

$$
\frac{1}{\tau_{n}}=\frac{1}{\tau_{b}}+D_{p} a_{s}^{2} n^{2}
$$

Let us denote the effective lifetime of minority carriers as $\tau_{\text {eff }}=\tau_{1}$ and the surface lifetime as $\tau_{s}=\left(D_{p} a_{s}^{2}\right)^{-1}$. The surface lifetime shows the effect of surface recombination on the effective lifetime of minority carriers.

Consider double-sided macroporous silicon, the cross-section of which is schematically shown in Fig. 1. It is a plate of monocrystalline silicon, in which, on each of the two largest parts in terms of area, macropores are etched. Let double-sided macroporous silicon be illuminated by light incident on one of the sides with macropores, parallel to macropores. A layer of macroporous silicon, on which light is incident, we call the input (for light) macroporous layer, while another macroporous layer let be called as the output macroporous layer. The input layer of macroporous silicon has the thickness equal to the depth of its macropores $h_{p o r 1}$ (see Fig. 1). 


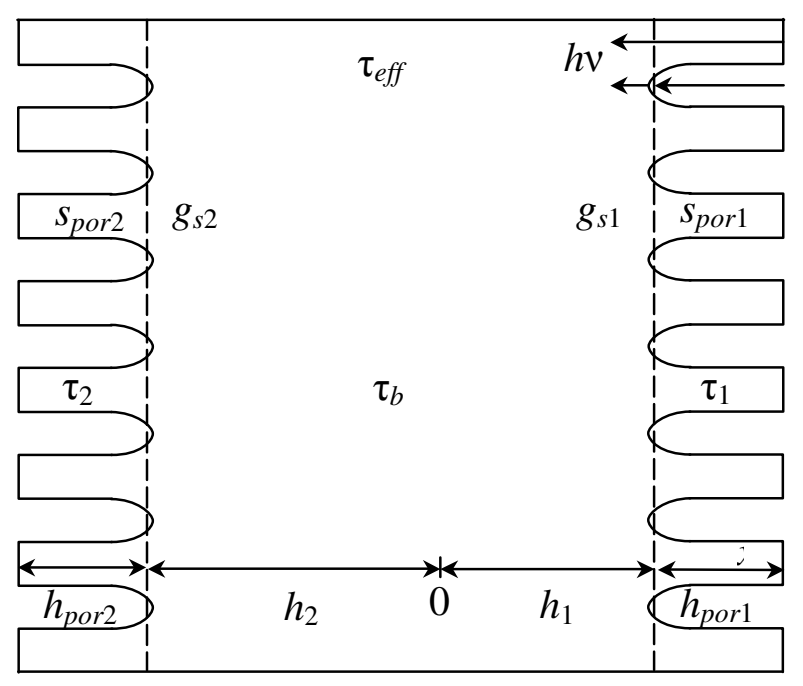

Fig. 1. Cross-section of double-sided macroporous silicon.

The output layer of macroporous silicon has the thickness equal to the depth of its macropores $h_{\text {por } 2}$. Between these two macroporous layers, there is a layer of monocrystalline silicon, which is a monocrystalline substrate. The bottom of the macropores of each of the macroporous layers is the surface of the monocrystalline layer.

Consider the distribution of the concentration of excess charge carriers in macroporous silicon. The concentration of excess charge carriers in the layer of macroporous silicon will decrease much more than in the monocrystalline substrate. The concentration gradient will arise, which will cause the diffusion of carriers from the substrate into the macroporous layer. As a result, in the monocrystalline part, the distribution function of excess minority charge carriers will have a maximum caused by the fact that in macroporous layers recombination is more intense due to recombination on the surface of macropores. Also, there will be a maximum in the input macroporous layer, which occurs at high values of light absorption coefficients [15]. But the maximum in the input macroporous layer will not be observed, provided that the distribution function of the excess minority charge carriers is described by the first terms of the Fourier series, according to the equation (3). The origin of the $x$-axis will be selected at the maximum point, as shown in Fig. 1. The $x$-axis is directed towards the input macroporous layer, parallel to the pores. Let us denote the coordinate of the point of the plane passing through the bottom of macropores in the incoming macroporous layer and perpendicular to the $x$-axis through $h_{1}$. Let us denote the coordinate of the point of the plane passing through the bottom of macropores in the output macroporous layer and perpendicular to the $x$ axis through $h_{2}$. The initial distribution functions of excess charge carriers differ in each layer of double-sided macroporous silicon. This difference is related with different illumination intensities, specificity of relaxation processes that occur in the bulk and on the surface of macropores, as well as diffusion velocities. From the equation (3), the distribution function of excess charge carriers in the input layer of macroporous silicon $\delta p_{1}(x)$, in the monocrystalline layer $\delta p_{m}(x)$ and in the output macroporous layer $\delta p_{2}(x)$ can be written as follows:

$$
\begin{aligned}
& \delta p_{1}(x, t)=A_{11} \exp \left(-\frac{t}{\tau_{e f f}}\right) \cos \left(a_{s 1} x\right), \\
& \delta p_{m}(x, t)=A_{1 m} \exp \left(-\frac{t}{\tau_{e f f}}\right) \cos \left(a_{s m} x\right), \\
& \delta p_{2}(x, t)=A_{12} \exp \left(-\frac{t}{\tau_{e f f}}\right) \cos \left(a_{s 2} x\right),
\end{aligned}
$$

where $A_{11}, A_{1 m}, A_{12}$ are the constant coefficients of the distribution function for excess charge carriers in the input macroporous layer, monocrystalline layer and output macroporous layer, respectively. The equations (5) to (7) are written under the condition that $t>>\tau_{\text {eff }}$ and only the first term remains in the equation (3). Relaxation of the distribution of excess charge carriers will occur exponentially, both in macroporous silicon and in a monocrystalline substrate with an effective relaxation time $\tau_{\text {eff. }}$ Let us consider the case when the depth of macropores is $h_{\text {por } 1} \gg L_{1}$, and $h_{\text {por } 2} \gg L_{2}$, where $L_{1}=\sqrt{D_{p} \tau_{1}}, L_{2}=\sqrt{D_{p} \tau_{2}}$ is the effective diffusion length of minority charge carriers in the first and second macroporous layers, respectively. We wrote down $\tau_{1}, \tau_{2}$ according to [15]:

$$
\begin{aligned}
& \frac{1}{\tau_{1}}=\frac{1}{\tau_{b}}+\frac{\pi D_{\text {por } 1} s_{\text {por } 1}}{a_{\text {por } 1}^{2}-0.25 \pi D_{\text {por } 1}^{2}}, \\
& \frac{1}{\tau_{2}}=\frac{1}{\tau_{b}}+\frac{\pi D_{\text {por } 2} s_{\text {por } 2}}{a_{\text {por } 2}^{2}-0.25 \pi D_{\text {por } 2}^{2}},
\end{aligned}
$$

where $D_{\text {por } 1}, D_{\text {por } 2}$ are the diameters of macropores in the input and output macroporous layers, respectively, $a_{\text {por } 1}$, $a_{\text {por } 2}$ are the average distances between the centers of the macropores in the input and output macropores layers, respectively, and $s_{\text {por } 1}, s_{\text {por } 2}$ are the rates of surface recombination on the surface of pores in the input and output macropores layers, accordingly.

In order for the distribution functions of excess minority charge carriers (5) to (7) to be equal at the boundary of the input or output macroporous layers with the monocrystalline layer, boundary conditions should be written in each plane passing through the surface of the monocrystalline layer (along the bottom of macropores). For any time value $t>\tau_{e f f}$, in each plane passing through the surface of the monocrystalline layer, the boundary condition (2) can be written in the following manner: 
$-\left(1-P_{1}\right) g_{s 1}\left(h_{1}, t\right)-P_{1} s_{p o r 1} \delta p_{m}\left(h_{1}, t\right)=-j_{m 1 p}\left(h_{1}, t\right)$,

$-\left(1-P_{2}\right) g_{s 2}\left(h_{2}, t\right)-P_{2} s_{p o r 2} \delta p_{m}\left(h_{2}, t\right)=-j_{m 2 p}\left(h_{2}, t\right)$,

where $g_{s 1}\left(h_{1}, t\right), g_{s 2}\left(h_{2}, t\right)$ correspond to surface generation of excess charge carriers in the plane passing through the bottom of macropores for the input and output surface of the monocrystalline layer, respectively, $P_{1}, P_{2}$ are the volume fractions of pores for the input and output layers of macroporous silicon, respectively, $j_{1 p}\left(h_{1}, t\right), j_{2 p}\left(h_{2}, t\right)$ are the diffusion current densities of excess minority charge carriers in the plane passing through the bottom of pores in the input and output layers of macroporous silicon, respectively. We put the minus sign before the value of the function corresponding to surface generation in the input and output planes of monocrystalline silicon, because in the plane passing through the bottom of the macropores, charge carriers that came from the monocrystalline layer disappear. The expression (11) is written as based on the symmetry of double-sided macroporous silicon with respect to the origin. Mentally divide the macroporous silicon at the origin. We have two similar pieces of macroporous silicon. Further, we will write the boundary conditions, as for the positive part of the coordinate line. With account of the expressions (5) and (7), the diffusion current density of excess minority charge carriers at the surface that passes through the input or output surfaces of monocrystalline silicon can be written as:

$$
\begin{aligned}
& j_{m 1 p}\left(h_{1}, t\right)=-D_{p} \frac{\partial}{\partial x} \delta p_{m}\left(h_{1}, t\right)= \\
& =D_{p} \delta p_{m}\left(h_{1}, t\right) a_{s m} \tan \left(a_{s m} h_{1}\right), \\
& j_{m 2 p}\left(h_{2}, t\right)=-D_{p} \frac{\partial}{\partial x} \delta p_{m}\left(h_{2}, t\right)= \\
& =D_{p} \delta p_{m}\left(h_{2}, t\right) a_{s m} \tan \left(a_{s m} h_{2}\right) .
\end{aligned}
$$

For any time value $t \gg \tau_{e f f}$, the boundary condition (2) for the input and output macroporous layer in the plane passing through the input or output surfaces of monocrystalline silicon, we can write as follows:

$g_{s 1}\left(h_{1}, t\right)=j_{1 p}\left(h_{1}, t\right)=-D_{p} \frac{\partial}{\partial x} \delta p_{1}\left(h_{1}, t\right)$

$g_{s 2}\left(h_{2}, t\right)=j_{2 p}\left(h_{2}, t\right)=-D_{p} \frac{\partial}{\partial x} \delta p_{2}\left(h_{2}, t\right)$

We did not take into account the surface recombination at the bottom of the macropores, since the bottom of the macropores is the surface of the monocrystalline substrate. We substitute the expressions (12), (13) into the expressions (10) and (14), and the expression (15) into the expression (11) and we write:

$$
\begin{aligned}
& \left(1-P_{1}\right) D_{p} \frac{\partial}{\partial x} \delta p_{1}\left(h_{1}, t\right)-P_{1} s_{p o r 1} \delta p_{m}\left(h_{1}, t\right)= \\
& =-D_{p} \delta p_{m}\left(h_{1}, t\right) a_{s m} \tan \left(a_{s m} h_{1}\right), \\
& \left(1-P_{2}\right) D_{p} \frac{\partial}{\partial x} \delta p_{2}\left(h_{2}, t\right)-P_{2} s_{p o r} \delta p_{m}\left(h_{2}, t\right)= \\
& =-D_{p} \delta p_{m}\left(h_{2}, t\right) a_{s m} \tan \left(a_{s m} h_{2}\right) .
\end{aligned}
$$

In the plane passing through the input or output surfaces of monocrystalline silicon, there should be no discontinuity of the function, that is $p_{1}\left(h_{1}, t\right)=p_{m}\left(h_{1}, t\right)$, $p_{m}\left(h_{2}, t\right)=p_{2}\left(h_{2}, t\right)$, therefore the equations (16) and (17) can be rewritten as:

$$
\begin{aligned}
& a_{s m} \tan \left(a_{s m} h_{1}\right)=\frac{1}{D_{p}}\left(P_{1} s_{p o r 1}-\frac{\frac{\partial}{\partial x} \delta p_{1}\left(h_{1}, t\right)}{\delta p_{1}\left(h_{1}, t\right)} D_{p}\left(1-P_{1}\right)\right), \\
& a_{s m} \tan \left(a_{s m} h_{2}\right)=\frac{1}{D_{p}}\left(P_{2} s_{p o r 2}-\frac{\frac{\partial}{\partial x} \delta p_{2}\left(h_{2}, t\right)}{\delta p_{2}\left(h_{2}, t\right)} D_{p}\left(1-P_{2}\right)\right) .
\end{aligned}
$$

We write the boundary conditions on two surfaces of the sample of macroporous silicon:

$$
\begin{aligned}
& s_{1} \delta p_{1}\left(h_{p o r 1}+h_{1}, t\right)=D_{p} \frac{\partial}{\partial x} \delta p_{1}\left(h_{p o r 1}+h_{1}, t\right), \\
& s_{2} \delta p_{2}\left(h_{p o r 2}+h_{2}, t\right)=D_{p} \frac{\partial}{\partial x} \delta p_{2}\left(h_{p o r 2}+h_{2}, t\right),
\end{aligned}
$$

where $s_{1}, s_{2}$ are the surface recombination rates on the surfaces of the sample of double-sided macroporous silicon, respectively. The systems of equations (4) to (9) and (18) to (21) describe the processes of relaxation of the distribution of excess minority charge carriers in double-sided macroporous silicon. Let us transform and simplify this system of equations. Eq. (3) is a general solution to Eq. (1). Provided that $t \gg \tau_{e f f}$, relaxation of excess charge carriers will occur exponentially as shown by Eqs (5) to (7). This means that we have a stationary distribution of the concentration function of excess minority carriers on the coordinate, which is constantly multiplied by exponent with the index $t$. The stationary solution of equation (1) multiplied by the exponent over time will be written for macroporous layers as follows:

$$
\begin{aligned}
& \delta p_{1}(x, t)=\exp \left(-\frac{t}{\tau_{\text {eff }}}\right)\left(C_{1} \exp \left(\frac{x}{L_{1}}\right)+C_{2} \exp \left(-\frac{x}{L_{1}}\right)\right), \\
& \delta p_{2}(x, t)=\exp \left(-\frac{t}{\tau_{\text {eff }}}\right)\left(C_{1^{*}} \exp \left(\frac{x}{L_{2}}\right)+C_{2^{*}} \exp \left(-\frac{x}{L_{2}}\right)\right),
\end{aligned}
$$

where $C_{1}, C_{2}, C_{1 *}, C_{2^{*}}$ are steel. We write the ratio of the derivative of the function from equations (22), (23) to the function itself at the points $h_{1}, h_{2}$ : 


$$
\begin{aligned}
& \frac{\frac{\partial}{\partial x} \delta p_{1}\left(h_{1}, t\right)}{\delta p_{1}\left(h_{1}, t\right)}=\frac{1}{L_{1}}\left(1-\frac{2}{\frac{C_{1}}{C_{2}} \exp \left(\frac{2 h_{1}}{L_{1}}\right)+1}\right), \\
& \frac{\frac{\partial}{\partial x} \delta p_{2}\left(h_{2}, t\right)}{\delta p_{2}\left(h_{2}, t\right)}=\frac{1}{L_{2}}\left(1-\frac{2}{\frac{C_{1^{*}}}{C_{2^{*}}} \exp \left(\frac{2 h_{2}}{L_{2}}\right)+1}\right) .
\end{aligned}
$$

Substitute the expressions (22) and (23) into the expressions (20) and (21), which describe the boundary conditions on two planes of the sample of macroporous silicon, and write:

$$
\begin{aligned}
& \frac{C_{1}}{C_{2}}=\exp \left(\frac{-2\left(h_{p o r 1}+h_{1}\right)}{L_{1}}\right)\left(\frac{2}{S_{1}+1}-1\right), \\
& \frac{C_{1^{*}}}{C_{2^{*}}}=\exp \left(\frac{-2\left(h_{p o r 2}+h_{2}\right)}{L_{2}}\right)\left(\frac{2}{S_{2}+1}-1\right),
\end{aligned}
$$

where $S_{1}=s_{1} L_{1} / D_{p}, S_{2}=s_{2} L_{2} / D_{p}$ are the dimensionless surface recombination velocities. Substitute the expressions (24) and (26) into the expression (18), and the expressions (25), (27) into the expression (19) and write:

$$
\begin{aligned}
& \tan \left(a_{s m} h_{1}\right)=\frac{1}{a_{s m} L_{1}} \times \\
& \times\left(P_{1} S_{p o r 1}+\left(1-P_{1}\right)\left(\frac{2}{\exp \left(\frac{-2 h_{\text {por } 1}}{L_{1}}\right)\left(\frac{2}{S_{1}+1}-1\right)+1}-1\right),\right.
\end{aligned}
$$

$$
\begin{aligned}
& \tan \left(a_{s m}\left(h-\left(h_{p o r 1}+h_{p o r 2}+h_{1}\right)\right)\right)=\frac{1}{a_{s m} L_{2}} \times \\
& \times\left(P_{2} S_{p o r 2}+\left(1-P_{2}\right)\left(\frac{2}{\exp \left(\frac{-2 h_{p o r} 2}{L_{2}}\right)\left(\frac{2}{S_{2}+1}-1\right)+1}-1\right)\right),
\end{aligned}
$$

where $S_{\text {por } 1}=s_{\text {por } 1} L_{1} / D_{p}, \quad S_{\text {por } 2}=s_{\text {por } 2} L_{2} / D_{p} \quad$ are the dimensionless surface recombination velocities on the pore surface of the first and second macroporous layer, respectively. The sum in brackets of the expression (29) is equivalent to the dimensionless surface recombination rate in the plane passing through the bottom of macropores. It consists of the dimensionless surface recombination rate on the surface of the pore (the first term in brackets) and the rate of flow of charge carriers from the monocrystalline substrate in the macroporous layer (the second term in brackets). We reduced the system of ten equations (4) to (9), (20) to (23) to two equations (28), (29) with two unknowns $a_{s m}$ and $h_{1}$. The constant characteristic number in the monocrystalline layer $a_{s m}$ is found from the system of equations (28), (29) and expressions (8), (9). Knowing $a_{s m}$, the effective lifetime of minority charge carriers is found from the expression (4), when $n=1$, which is written as:

$\frac{1}{\tau_{e f f}}=\frac{1}{\tau_{b}}+D_{p} a_{s m}^{2}$.

In the absence of an output layer of macroporous silicon $P_{2}=0, h_{\text {por } 2}=0, S_{2}=0$, the expression (29) becomes the expression for a single crystal and the expressions (28), (29) - to define the efficient lifetime of minority carriers in macroporous silicon with one macroporous layer. In the absence of input and output layers of macroporous silicon $P_{1}=0, h_{\text {por } 1}=0, S_{1}=0, P_{2}=0, h_{\text {por } 2}=0, S_{2}=0$, then the expressions (28), (29) define the effective lifetime of minority carriers in a single crystal.

\section{Calculation of the effective lifetime of minority charge carriers}

Shown in Figs $2 \mathrm{a}$ to $2 \mathrm{f}$ is the dependence of the effective lifetime of minority charge carriers in one-sided (Fig. 2f) and double-sided macroporous silicon on the depth of macropores. The effective lifetime of minority carriers was calculated using the expressions (8), (9), (28), (29) and (30). For these calculations, the following data were used: the bulk lifetime equal to $10 \mu \mathrm{s}$, surface recombination velocity on all the surfaces was the same and equal to $0.9 \mathrm{~m} / \mathrm{s}$, the average diameter of macropores was $2 \mu \mathrm{m}$, the average distance between the centers of macropores was $4 \mu \mathrm{m}$. The value $\tau_{1}=\tau_{2}=1 \mu \mathrm{s}$ was calculated using the expressions (8), (9). The thickness of the sample of macroporous silicon is $500 \mu \mathrm{m}$. The thickness of the output macroporous layer changed by $100 \mu \mathrm{m}$ and was also equal to $10 \mu \mathrm{m}$ and was absent in one-sided macroporous silicon. If the right-hand side of expressions (28) and (29) is less than one, then the effective lifetime of minority carriers is determined by the surface recombination velocity on the surface of macropores in the sample. With an increase in the depth of macropores, recombination in the layer of macroporous silicon greatly increases due to an increase in the recombination surfaces on the pores. Due to this, the effective lifetime of minority charge carriers sharply decreases with increasing the macropore depth in the input macroporous silicon (Figs 2a-2f) and output macroporous silicon (abrupt transition from curve of Fig. 2f, when $h_{\text {por } 2}=0$, to curve of Fig. 2e, when $\left.h_{\text {por } 2}=10 \mu \mathrm{m}\right)$. That is, for non-deep macropores, the effective lifetime of minority charge carriers depends on the bulk lifetime and surface recombination in the macroporous layer, which is defined by the depth of the macropores, with a constant thickness of the sample. If the right-hand side of expressions (28) and (29) is higher than unity, due to the large dimensionless velocity of surface recombination, or to an expression with an 


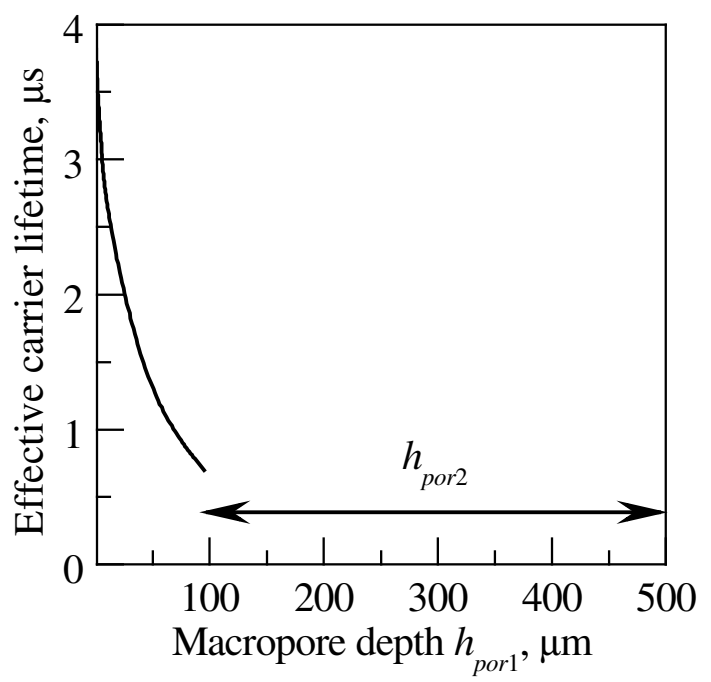

a)

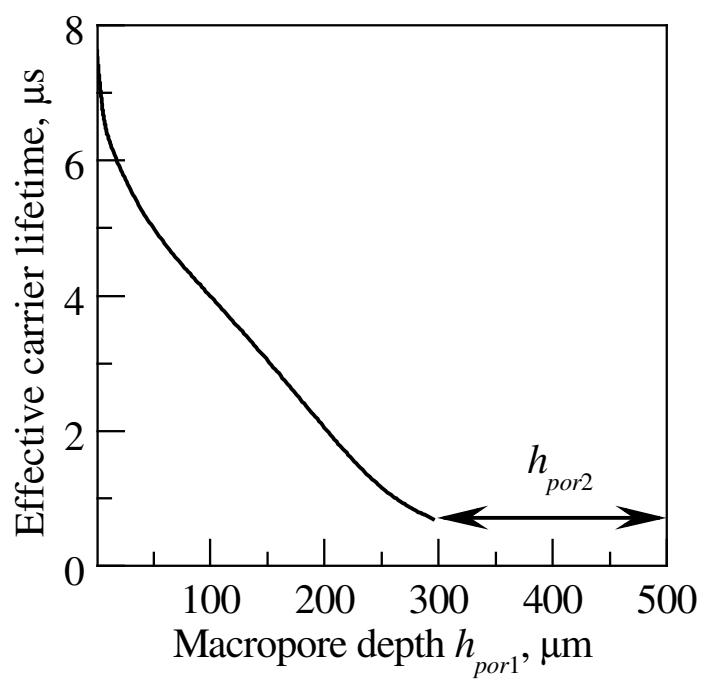

c)

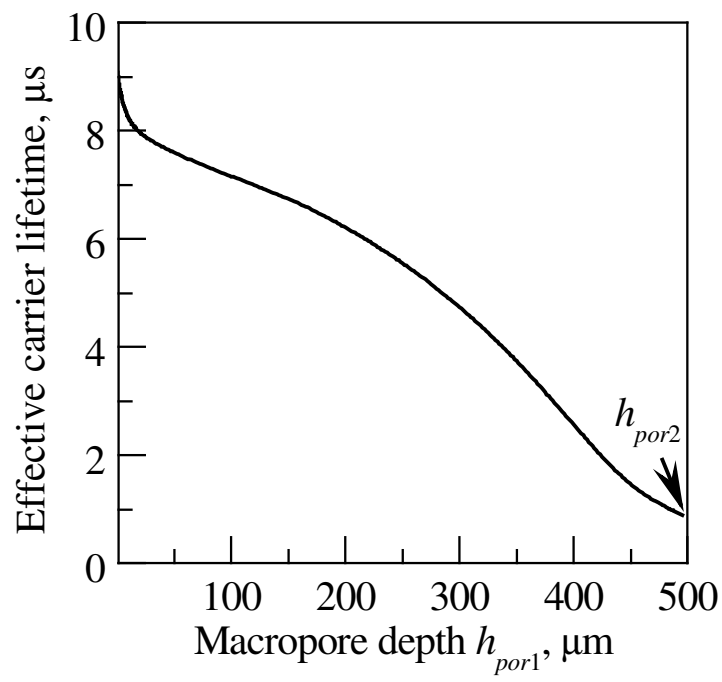

e)

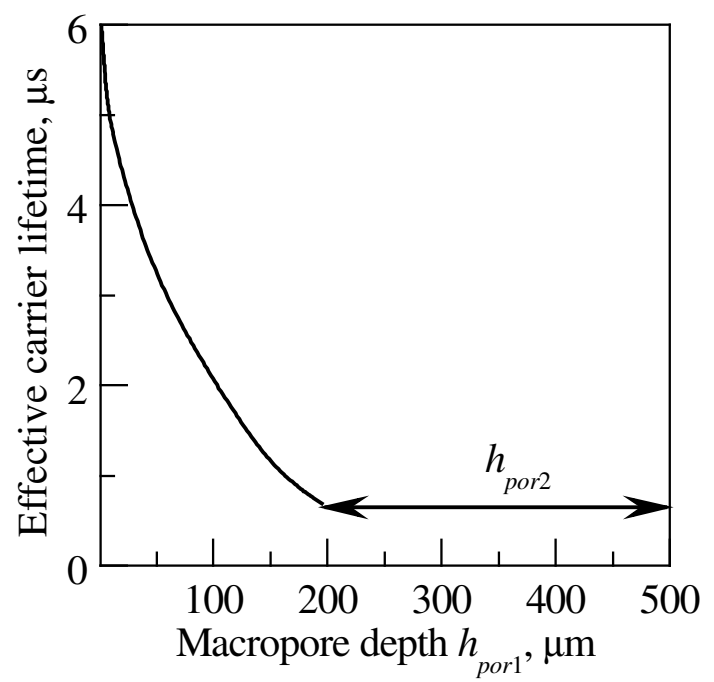

b)

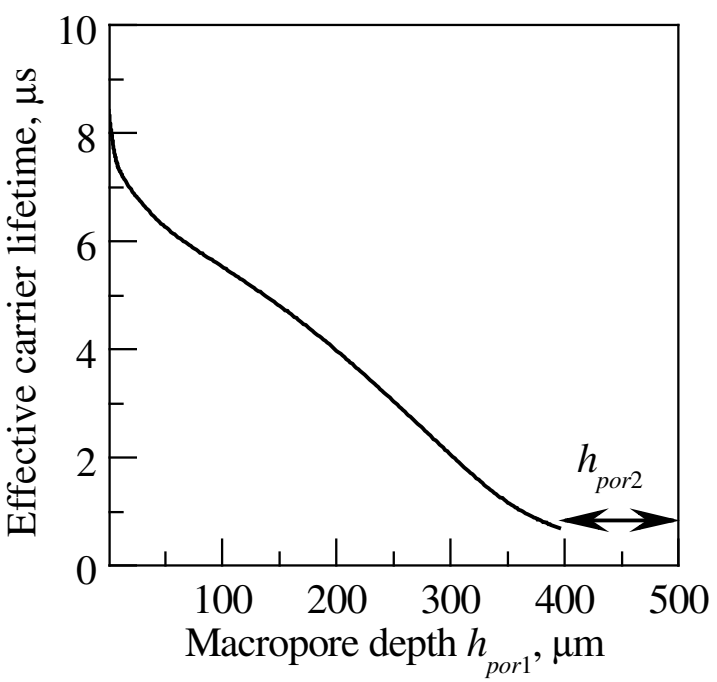

d)

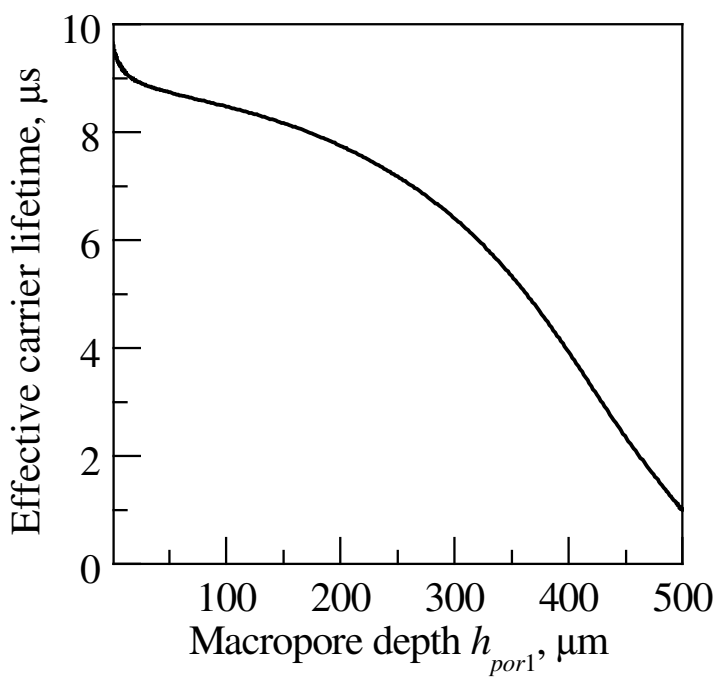

f)

Fig. 2. The efficient lifetime of minority carriers in one-sided $\left(f, h_{p o r 2}=0\right)$ and double-sided macroporous silicon, depending on the depth of macropores in the input macroporous layer, which is calculated for the depths of macropores in the output macroporous layer, $\mu \mathrm{m}$ : (a) -400 , (b) - 300, (c) - 200, (d) - 100, (e) - 10, (f) -0 . 
exponent, then we can write approximately $a_{s m} h_{1} \approx \pi / 2$, $a_{\text {sm }}\left(h-\left(h_{\text {por } 1}+h_{\text {por } 2}+h_{1}\right)\right) \approx \pi / 2$. If the right-hand side of expressions (28) and (29) is higher than unity, then the effective lifetime of minority carriers from the expression (30) can be written as follows:

$$
\frac{1}{\tau_{e f f}} \approx \frac{1}{\tau_{b}}+\frac{\pi^{2} D_{p}}{\left(h-h_{p o r 1}-h_{p o r 2}\right)^{2}} .
$$

In this case, the effective lifetime of minority carriers in double-sided macroporous silicon does not depend on the surface recombination velocity, despite the fact that it is high on the surface of macropores. The effective lifetime depends on the bulk lifetime, has a quadratic dependence on the thickness of the monocrystalline substrate, which depends on the depth of macropores, and is inversely proportional to the diffusion coefficient. As can be seen from Figs $2 \mathrm{a}$ to $2 \mathrm{c}$, the effective lifetime of minority carriers has a quadratic dependence on the depth of macropores. This is due to the fact that the bulk lifetime can be neglected.

Otherwise, as can be seen from Figs $2 d$ to $2 e$, the bulk lifetime cannot be neglected. Thus, the surface recombination velocity in macroporous silicon layers increases with increasing macropore area, but when it becomes very high, then recombination at the pore surface is limited by the diffusion of charge carriers from the monocrystalline substrate and the thickness of the monocrystalline substrate. Excess carriers have no time to diffuse to the recombination surfaces of macroporous silicon layers; therefore, effective recombination of excess charge carriers does not depend on recombination in macroporous silicon layers. In this case, with increasing the depth of the macropores and the area of the recombination surfaces, recombination in the macroporous layer does not change. The dependence of effective lifetime of minority carriers on the depth of macropores in the input macroporous silicon in one-sided and double-sided macroporous silicon is similar, as can be seen from Figs $2 \mathrm{e}$ and $2 \mathrm{f}$. The appearance of macropores on the other side of one-sided macroporous silicon leads to a sharp decrease in the effective lifetime due to additional recombination in the initial macroporous silicon.

\section{Conclusion}

The effective lifetime of minority charge carriers in the double-sided macroporous silicon is defined from a system of two equations. The first equation describes the processes in the input macroporous layer and at the boundary "input macroporous layer - monocrystalline substrate". The second equation describes the processes in the output macroporous layer and at the interface "output macroporous layer - monocrystalline substrate".

It has been found that the effective lifetime of minority carriers in the double-sided macroporous silicon depends on the bulk lifetime of minority carriers, charge carrier diffusion coefficient, monocrystalline substrate thickness, and parameters of each macroporous layer: the average macropore diameter, average distance between centers of macropores, surface recombination velocity, volume fraction of macropores.

At a small depth of macropores, when $\tan \left(a_{s m} h_{1}\right)>>1$ or $\tan \left(a_{s m}\left(h-\left(h_{\text {por } 1}+h_{\text {por } 2}+h_{1}\right)\right)\right)>>1$

from Eqs (28), (29), the effective lifetime of minority carriers depends on bulk lifetime and surface recombination in the macroporous layer, that is, it is defined by the depth of macropores, with a constant thickness of the sample.

At a high surface recombination velocity on the surface of macropores, when $\tan \left(a_{s m} h_{1}\right)>>1$ or $\tan \left(a_{s m}\left(h-\left(h_{\text {por } 1}+h_{\text {por } 2}+h_{1}\right)\right)\right)>>1$ from Eqs (28), (29), the effective lifetime of minority carriers depends on the bulk lifetime and has a quadratic dependence on the thickness of the monocrystalline substrate, which depends on the depth of the macropores, and inversely depends on the diffusion coefficient.

When the surface recombination velocity in macroporous silicon layers becomes very high $\tan \left(a_{s m} h_{1}\right)>>1$ or $\tan \left(a_{s m}\left(h-\left(h_{\text {por } 1}+h_{\text {por } 2}+h_{1}\right)\right)\right)>>1$, then recombination on the pore surface is limited by diffusion of charge carriers from the monocrystalline substrate and the thickness of this substrate. Excess charge carriers have no time to diffuse to the recombination surfaces of macroporous layers, therefore, effective recombination of excess charge carriers does not depend on recombination in macroporous layers, in this case, with an increase in the depth of macropores and the area of the recombination surfaces, recombination in the macroporous layer does not change.

The dependence of effective lifetime for minority carriers on the depth of macropores of the input macroporous silicon in one-sided and double-sided macroporous silicon is similar. The appearance of macropores on the other side of one-sided macroporous silicon leads to a sharp decrease in the effective lifetime due to additional recombination in the output macroporous silicon.

It has been shown that the effective lifetime of minority charge carriers in the double-sided macroporous silicon is limited by diffusion of charge carriers from the substrate to recombination surfaces in each macroporous layer and has a quadratic dependence on the thickness of the monocrystalline substrate.

\section{References}

1. Ernst M., Brendel R., Ferre R., Harder N.P. Thin macroporous silicon heterojunction solar cells. phys. status solidi RRL. 2012. 6, No 5. P. 187-189. https://doi.org/10.1002/pssr.201206113.

2. Ernst M. and Brendel R. Macroporous silicon solar cells with an epitaxial emitter. IEEE J. Photovolt. 2013. 3, No 2. P. 723-729. https://doi.org/10.1109/JPHOTOV.2013.2247094. 
3. Selj J.H., Marstein E., Thogersen A., Foss S.E. Porous silicon multilayer antireflection coating for solar cells; process considerations. phys. status solidi C. 2011. 8, No 6. P. 1860-1864.

https://doi.org/10.1002/pssc.201000033.

4. Maiolo J.R., Atwater H.A., Lewis N.S. Macroporous silicon as a model for silicon wire array solar cells. J. Phys. Chem. C. 2008. 112, No 15. P. 6194-6201.

https://doi.org/10.1021/jp711340b.

5. Karachevtseva L.A., Lytvynenko O.O., Konin K.P., Parshyn K.A., Sapelnikova O.Yu., Stronska O.J. Electro-optical effects in 2D macroporous silicon structures with nanocoatings. Semiconductor Physics Quantum Electronics \& Optoelectronics. 2015. 18, No 4. P. 377-384.

https://doi.org/10.15407/spqeo18.04.377.

6. Evtukh A.A., Litovchenko V.G., Klyui N.I., Semenenko M.O., Kaganovich E.B., Manoilov E.G. Electron field emission from porous silicon prepared at low anodisation currents. International Journal of Nanotechnology. 2006. 3, No 1, P. 89105.

https://doi.org/10.1504/IJNT.2006.008723.

7. Ernst M., Brendel R. Modeling effective carrier lifetimes of passivated macroporous silicon layers. Solar Energy Materials and Solar Cells. 2011. 95, No 4. P. 1197-1202.

https://doi.org/10.1016/j.solmat.2011.01.017.

8. Onyshchenko V.F., Karachevtseva L.A., Lytvynenko O.O., Plakhotnyuk M.M., Stronska O.Y. Effective lifetime of minority carriers in black silicon nano-textured by cones and pyramids. Semiconductor Physics, Quantum Electronics \& Optoelectronics. 2017. 20, No 3. P. 325-329. https://doi.org/10.15407/spqeo20.03.325.

9. Ivanov V.I., Karachevtseva L.A., Karas N.I., Lytvynenko O.A., Parshin K.A., Sachenko A.V. Photoconductivity in macroporous silicon with regular structure of macropores. Semiconductor Physics Quantum Electronics \& Optoelectronics. 2007. 10, No 4. P. 72-76.

10. Karachevtseva L.A., Karas' M.I., Onishchenko V.F., Sizov F.F. Enhancement of the photoconductivity in 2D photonic macroporous silicon structures. Photonic Crystal Materials and Devices II, Proc. SPIE. 2004. 5360. P. 381-389. https://doi.org/10.1117/12.530446.

11. Karachevtseva L.A., Onyshchenko V.F., Sachenko A.V. Photoconductivity relaxation and electron transport in macroporous silicon structures. Semiconductor Physics, Quantum Electronics \& Optoelectronics. 2017. 20, No 4. P. 475-480. https://doi.org/10.15407/spqeo20.04.475.

12. Evtukh A.A., Kaganovich E.B., Manoilov E.G., Semenenko N.A. A mechanism of charge transport in electroluminescent structures consisting of porous silicon and single-crystal silicon. Semiconductors. 2006. 40, No 2. P. 175-179. https://doi.org/10.1134/S1063782606020126.
13. Karachevtseva L., Onyshchenko V., and Sachenko A. Photocarrier transport in 2D macroporous silicon structures. Opto-Electronics Review. 2010. 18, No 4, P. 394-399. https://doi.org/10.2478/s11772-010-0042-7.

14. Onyshchenko V.F. Karachevtseva L.A. Effective minority carrier lifetime and distribution of steadystate excess minority carriers in macroporous silicon. Him. Fiz. Tehnol. Poverhni. 2017. 8, No 3. P. 322-332. https://doi.org/10.15407/hftp08.03.322.

15. Karachevtseva L.A., Onyshchenko V.F. Relaxation of excess minority carrier distribution in macroporous silicon. Him. Fiz. Tehnol. Poverhni. 2018. 9, No 2. P. 158-166.

https://doi.org/10.15407/hftp09.02.158.

\section{Authors and CV}

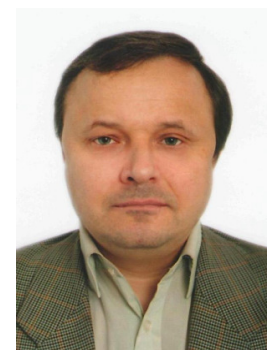

Onyshchenko V.F., Senior Researcher of the Laboratory of Photonic Semiconductor Structures at the V. Lashkaryov Institute of Semiconductor Physics. His main research interests include modelling photoconductivity kinetics in macroporous silicon and black silicon.

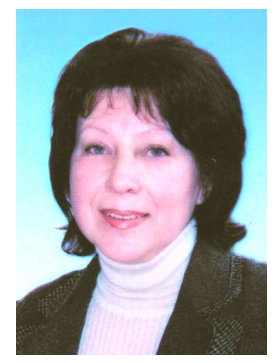

Karachevtseva L.A. Doctor of Technical Sciences, Head of the Laboratory of Photonic Semiconductor Structures at the V. Lashkaryov Institute of Semiconductor Physics. She is the author of more than 250 scientific publications. The area of her scientific interests includes photonic semiconductor structures,

photoelectrochemistry, semiconductor physics, transport phenomena and recombination. 